\title{
ATOMIC, MOLECULAR, AND OPTICAL PHYSICS
}

\section{RAPID COMMUNICATIONS}

The Rapid Communications section is intended for the accelerated publication of important new results. Since manuscripts submitted to this section are given priority treatment both in the editorial office and in production, authors should explain in their submittal letter why the work justifies this special handling. A Rapid Communication should be no longer than 4 printed pages and must be accompanied by an abstract. Page proofs are sent to authors.

\section{Observation of double-resonant laser-induced transitions in the $v=n-l-1=2$ metastable cascade of antiprotonic ${ }^{4} \mathrm{He}$ atoms}

\author{
R. S. Hayano, T. Ishikawa, H. Tamura, H. A. Torii, M. Hori, and F. E. Maas*
}

Department of Physics, University of Tokyo 7-3-1 Hongo, Bunkyo-ku, Tokyo 113, Japan

N. Morita and M. Kumakura

Institute for Molecular Science, Myodaiji, Okazaki 444, Japan

I. Sugai

Institute for Nuclear Study, University of Tokyo, 3-2-1 Midori-cho, Tanashi, Tokyo 188, Japan

F. J. Hartmann, H. Daniel, T. von Egidy, B. Ketzer, and R. Pohl

Physik Department, Technische Universität München, D-85747 Garching, Germany

D. Horváth

KFKI Research Institute for Particle and Nuclear Physics, H-1525 Budapest, Hungary

J. Eades, E. Widmann, and T. Yamazaki

CERN, CH-1211 Geneva 23, Switzerland

(Received 12 September 1996)

\begin{abstract}
Another laser-induced resonant transition in the $v=n-l-1=2$ metastable cascade of antiprotonic ${ }^{4} \mathrm{He}$ atoms has been found by using a double-resonance technique. This was done by setting the first laser to the already known $470.724-\mathrm{nm}$ resonance $[(n, l)=(37,34) \rightarrow(36,33)]$, while the $(38,35) \rightarrow(37,34)$ transition was searched for with the second laser. The resonant transition was found at a wavelength of $529.622 \pm 0.003 \mathrm{~nm}$, showing excellent agreement with a recent prediction of Korobov. [S1050-2947(97)50601-9]
\end{abstract}

PACS number(s): $36.10 . \mathrm{Gv}$

Stopped antiprotons live in matter usually only about $10^{-12} \mathrm{~s}$. An exceptional case is $\mathrm{He}$, where about $3 \%$ of the incoming stopped antiprotons live as long as about $\sim 3 \mu \mathrm{s}$ [1-4]. The longevity is due to antiprotons caught in high Bohr orbits with high angular momentum where only slow radiative transitions are allowed. We report here the observation of a laser-induced resonant transition between two metastable states of antiprotonic helium atoms $\left(\bar{p}^{4} \mathrm{He}^{+}\right)$

\footnotetext{
*Present address: Institut für Kernphysik, Universität Mainz, D-55099 Mainz, Germany.
}

[1-4]. These were formed by stopping antiprotons from the CERN Low Energy Antiproton Ring (LEAR) in a lowtemperature helium gas target $(4-6 \mathrm{~K}, \sim 0.5 \mathrm{bar})$. The resonance was detected by a technique using two laser beams fired simultaneously. We set one of these at the 470.724-nm wavelength of the known $(n, l)=(37,34)$ $\rightarrow(36,33)$ transition with vibrational quantum number $v=n-l-1=2$ and sought with the other near the theoretically expected wavelength of the $(38,35) \rightarrow(37,34)$ metastable-metastable transition immediately above it in the $v=2$ sequence.

The lower transition, being the last one in the $v=2$ cascade (see Fig. 1), proceeds from a metastable to a nonmeta- 


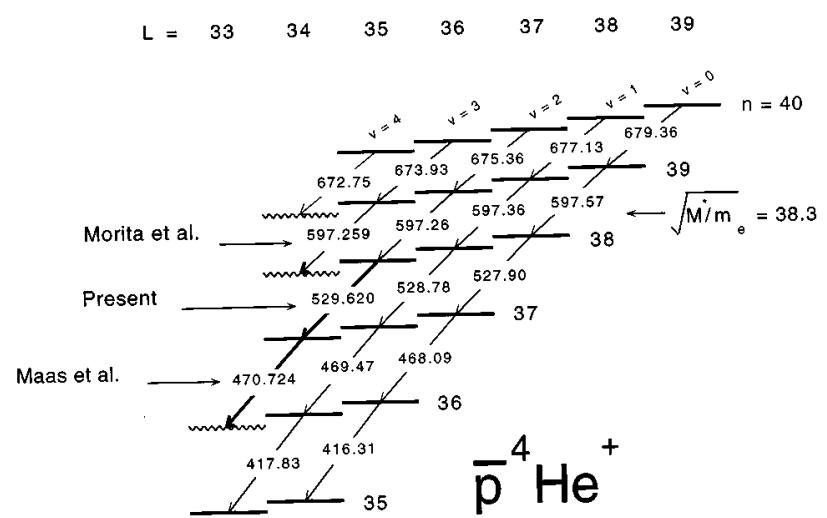

FIG. 1. Energy-level diagram of the $\bar{p}^{4} \mathrm{He}^{+}$atom. The transitions found in our previous work and the one found in the present double-resonance method are shown by thick arrows, with the observed wavelengths. All other transition wavelengths are taken from Korobov [12]. Metastable levels are indicated by solid lines and Auger-dominated short-lived states are drawn as wavy lines.

stable state. The $\sim 1 \mu$ s lifetime of the upper level thus ensured that it retained a large fraction of its initial population until the laser pulse arrived $\sim 1.7 \mu$ s after the atom was formed. However, the lower level was constantly being emptied by an Auger transition to $\bar{p}{ }^{4} \mathrm{He}^{2+}$ with lifetime $\tau \leqslant 10$ ns. The laser light could therefore effect a substantial net transfer of $\bar{p}$ to the $(36,33)$ state. As in our original observation of this transition [5], the extreme instability of the latter against annihilation via collisional Stark mixing [6,7] then produced a sharp peak, synchronous with the laser pulse, in the $\bar{p}$ annihilation rate. This peak served as a sensitive indicator of the resonance condition.

Clearly this single-laser technique will not work if the upper and lower levels are both metastable, since at any given instant their populations can be expected to be almost equal. In the present case, however, as the second laser was tuned through the upper transition frequency, antiprotons in the $(38,35)$ state were transferred through the $(37,34)$ level, already being drained by the first laser, to the $(36,33)$ level. The upper resonant condition then revealed itself as an increase in the height of the above-mentioned 470.724-nm annihilation peak.

Besides the 470.724-nm transition, we had previously observed another metastable-nonmetastable resonance at $597.259 \mathrm{~nm}$. We were able to assign this to the $v=3$ $(39,35) \rightarrow(38,34)$ sequence $([8,9])$ with the help of theoretical calculations of wavelengths and Auger lifetimes. These imply that only five metastable-nonmetastable transitions (those with $v=0,1,2,3,4)$ exist, and that only three of these $(v=2,3,4)$ are within easy reach of our visible-light dye laser system. The work described here, carried out with an experimental arrangement identical to that described in [8], except for the addition of the second laser system, is evidently an important step in extending our laser spectroscopy technique beyond these few transitions.

In reporting [5] the observation of the 470.724-nm transition we had, by varying the ignition times of two lasers at the same wavelength, already shown that the upper $(37,34)$ level population was being replenished from one or more still higher-lying states. This was deduced from the fact that
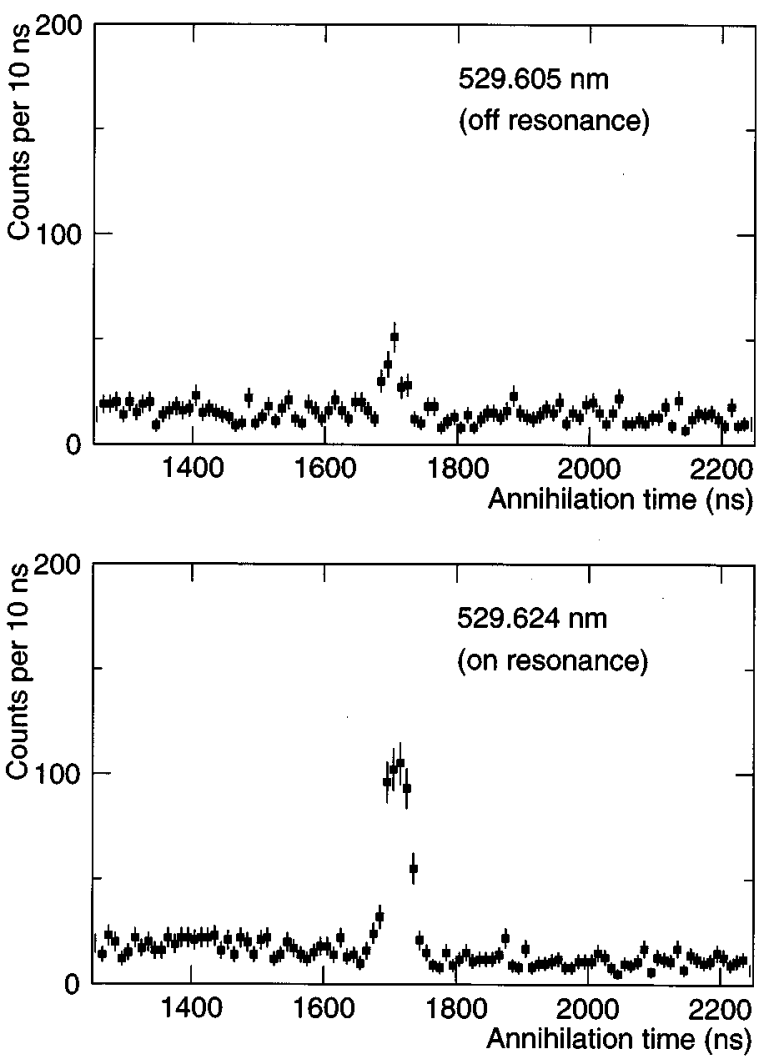

FIG. 2. Delayed annhilation time spectra taken with the singly resonant condition (upper panel: first laser at $470.724 \mathrm{~nm}$, second laser at $529.605 \mathrm{~nm}$ ) and the double-resonant condition (lower panel: first laser at $470.724 \mathrm{~nm}$, second laser at $529.622 \mathrm{~nm}$ ).

when the earlier-firing laser was used to empty the $(37,34)$ state, the later-firing one produced a second annihilation peak, indicating that the $(37,34)$ population had partially recovered in the meantime. Our usual assumption is that the radiative transitions between metastable states occur preferentially along the constant- $v$ cascade chain. In this case, the $(37,34)$ state must have been replenished from the $(38,35)$, possibly via still higher $v=2$ levels. A model based on this supposed chain decay sequence [5] gave an initial population of the $(38,35)$ state $\sim 5 \%$ of the total delayed fraction. We therefore expected that the $(38,35)$ state would still be populated at the time of arrival of the laser pulse, and that this would produce a net enhancement of the lower resonance peak.

Until very recently, theoretically calculated wavelengths for the $(n, l) \rightarrow(n-1, l-1)$ transition were all subject to uncertainties of order $1 \mathrm{~nm}[10,11]$. Korobov [12] has now used a molecular-expansion variational calculation that gives transition energies for the two already observed transitions within $50 \mathrm{ppm}$ of their measured values (i.e., $<0.03 \mathrm{~nm}$, only about five times the laser bandwidth). The high accuracy of Korobov's prediction already led us to a successful observation of laser-resonant transition in ${ }^{3} \mathrm{He}$ [13]. We therefore fixed the wavelength of one laser to the 470-nm resonance (blue), and scanned the second one around Korobov's predicted value of $529.60 \mathrm{~nm}$ (green); as in our previous measurements, the $\bar{p}$ annihilation time spectra were accumulated by detecting the annihilation pions. The pulses 

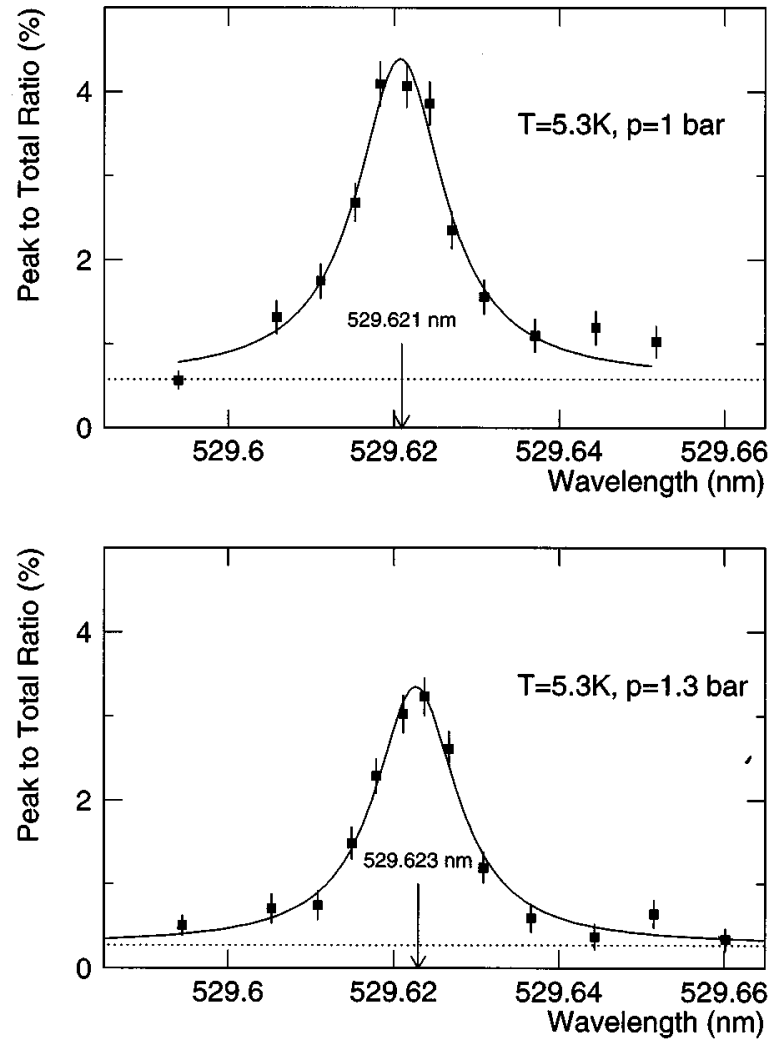

FIG. 3. Dependence of the double-resonance intensity on the wavelength of the second laser. The upper panel shows the resonance scan result taken with a helium gas target pressure of 1 bar and temperature of $5.3 \mathrm{~K}$, while the lower panel is for a pressure of 1.3 bar and temperature of $5.3 \mathrm{~K}$.

that ignited the two lasers were synchronized to within $\sim 5$ $\mathrm{ns}$, although the unavoidable time jitter of the excimer lasers that pump the tunable dye lasers caused the actual light pulse arrival time to vary with a full width at half maximum of $\sim 15 \mathrm{~ns}$. However, this jitter was well within the width of the light pulse ( 30-40 ns), so that the two laser pulses almost always overlapped in time.

The expected enhancement of the lower $(38,35)$ $\rightarrow(37,34)$ transition was soon found at an upper wavelength of $529.622 \mathrm{~nm}$. This can be seen in Fig. 2, which compares the annihilation time spectra with the second laser off and on resonance (upper and lower panels). The dependence of the annihilation peak counts (normalized to the total number of delayed events) on the second laser wavelength is shown in Fig. 3. The upper and lower panels show the scan result for helium at 1.0 bar and 1.3 bar, respectively, the temperature being in both cases $5.3 \mathrm{~K}$. The central wavelength of the resonant transition was found to be $529.622 \pm 0.003 \mathrm{~nm}$, where the errors cover both statistical errors and calibration errors for the wavelength meter.

In addition to the discovery of a new resonant transition, a closer look at Fig. 3 reveals the following interesting facts.

(i) The central wavelengths are slightly different for the two scans taken at different target pressures. They are $529.621 \mathrm{~nm}$ at 1 bar (upper panel) and $529.623 \mathrm{~nm}$ at $1.3 \mathrm{bar}$ (lower panel). This is a small but statistically significant difference, although we cannot draw a definitive conclusion

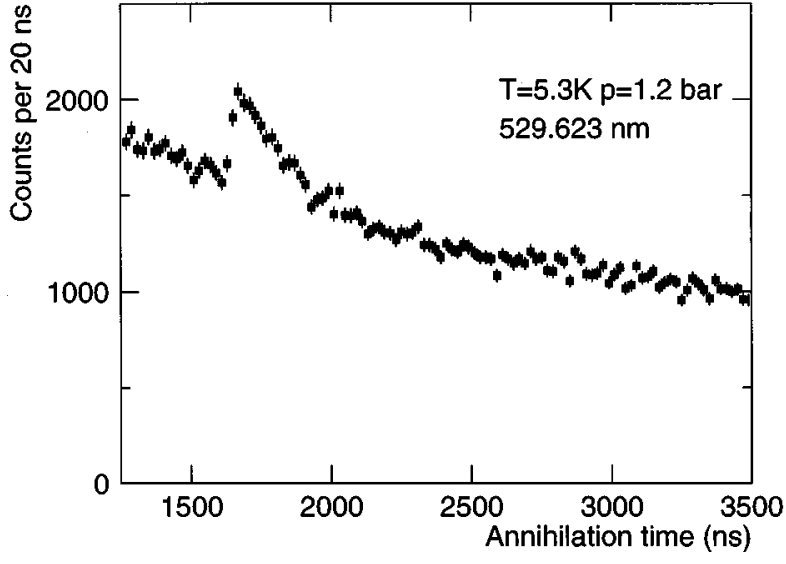

FIG. 4. Delayed annihilation time spectrum taken without the first laser and with the second laser set at the 529-nm resonance. The helium target was at a temperature of $5.3 \mathrm{~K}$ and pressure of 1.2 bar. At this pressure, the lifetime of $(37,34)$ level was about $\sim 0.2 \mu \mathrm{s}$, so that the $529-\mathrm{nm}$ resonance was visible without the aid of the first laser.

based on the measurements taken at only two pressure points. We later studied the pressure dependence of the resonance wavelengths both for the 470- and the 597-nm reso nances, and found that the wavelengths became longer as the pressure was increased, and that the pressure shift was state dependent. This will be discussed in detail in our forthcoming presentation [14].

(ii) The resonance peak heights also appear to have a pressure dependence. This is true for the 529-nm resonance, and also for the $470-\mathrm{nm}$ resonance. The latter can be seen by comparing the off-resonance peak to total ratio (indicated by the dotted lines in Fig. 3), which is about $0.6 \%$ at 1 bar and about $0.3 \%$ at 1.3 bar. These values are significantly smaller than the peak to total ratio of about $4 \%$ at 0.6 bar, reported in our previous publication [5]. We found that this is due to the strong pressure dependence of the lifetime of the $(37,34)$ level $\left(\tau_{37}\right)$. At low $(<0.2$ bar $)$ pressure, $\tau_{37}$ approached the calculated radiative-decay value of $1.37 \mu$ s, while it became as short as $\sim 0.3 \mu \mathrm{s}$ at 1 bar. A detailed account of the pressure dependence of the level lifetimes will be given elsewhere [15].

We note that the $(37,34)$ level is already being constantly drained at high pressure, so that the 529-nm resonance must be observable without the aid of the first laser. This we verified by turning off the first laser, and setting the second laser on resonance. A typical result taken at a pressure of 1.2 bar is shown in Fig. 4. The laser-induced annihilation peak is clearly visible, but unlike other cases the peak has a long tail, whose slope is determined by the lifetime of $(37,34)$ level at this pressure. In conclusion, we discovered a laser-induced resonant transition at $529.623 \mathrm{~nm}$ by introducing the double-resonance method, and ascribed it to the $(n, l)=(38,35) \rightarrow(37,34)$ transition in the $v=n-l-1=2$ metastable cascade.

We are indebted to the LEAR and PS staffs at CERN for their tireless dedication to providing us with the antiproton beam, and to V.I. Korobov for making his theoretical results 
available to us. The present work is supported by the Grantsin-Aid for Specially Promoted Research and for International Scientific Research of the Japanese Ministry of Education, Science and Culture; the German Bundesministerium für Bil- dung, Wissenchaft, Forschung und Technologie; and the Hungarian National Science Foundation. H.A.T. acknowledges the support of Japan Society for the Promotion of Science.
[1] M. Iwasaki et al., Phys. Rev. Lett. 67, 1246 (1991).

[2] T. Yamazaki et al., Nature (London) 361, 238 (1993).

[3] S. N. Nakamura et al., Phys. Rev. A 49, 4457 (1994).

[4] E. Widmann et al., Phys. Rev. A 51, 2870 (1995).

[5] F. E. Maas et al., Phys. Rev. A 52, 4266 (1995).

[6] N. Morita, K. Ohtsuki, and T. Yamazaki, Nucl. Instrum. Methods Phys. Res. A 330, 439 (1993).

[7] K. Ohtsuki (private communication).

[8] N. Morita et al., Phys. Rev. Lett. 72, 1180 (1994).
[9] R. S. Hayano et al., Phys. Rev. Lett. 73, 1485 (1994); 73, 3181(E) (1994).

[10] T. Yamazaki and K. Ohtsuki, Phys. Rev. A 45, 7782 (1992).

[11] I. Shimamura, Phys. Rev. A 46, 3776 (1992); and private communication.

[12] V.I. Korobov, Phys. Rev. A 54, 1749 (1996).

[13] H. A. Torii et al., Phys. Rev. A 53, R1931 (1996).

[14] H. A. Torii et al. (unpublished).

[15] M. Hori et al. (unpublished). 